\title{
Neutron stars in general relativity and scalar-tensor theory of gravity
}

Received: 2 April 2019 / Accepted: 28 June 2019 / Published online: 11 July 2019

(C) The Author(s) 2019

\begin{abstract}
The masses and radii of neutron stars are discussed in general relativity and scalar-tensor theory of gravity and the differences are compared with the current uncertainties stemming from the nuclear equation of state in the relativistic mean-field framework. It is shown that astrophysical and gravitational wave observations of radii of neutron stars with masses $M \lesssim 1.4 M_{\odot}$ constrain only the nuclear equation of state, and in particular the density dependence of the nuclear symmetry energy. Future observations of massive neutron stars may constrain the coupling parameters of the scalar-tensor theory provided that a general consensus on the dense nuclear matter equation of state is reached.
\end{abstract}

Mathematics Subject Classification (2000) $\quad 83 \mathrm{C} 05 \cdot 83 \mathrm{C} 35 \cdot 85 \mathrm{~A} 15 \cdot 53 \mathrm{Z} 05 \cdot 83 \mathrm{C} 99 \cdot 81 \mathrm{~V} 35 \cdot 83 \mathrm{D} 05 \cdot$ $83 \mathrm{C} 55$

\section{Introduction}

Neutron stars are ideal astrophysical laboratories to probe the nature of nuclear matter under extreme conditions of density and isospin asymmetry $[50-52,86,88]$ as well as to test fundamental theories of strong-field gravity $[72,97,100]$. While there has been significant improvement in understanding properties of dense nuclear matter at and near nuclear saturation density, $\rho_{0} \simeq 2.5 \times 10^{14} \mathrm{~g} / \mathrm{cm}^{3}$, our knowledge of dense matter at supersaturation densities $\rho>\rho_{0}$ corresponding to the core region of neutron stars remains quite poor. In part, it is related due to the fact that current nuclear interaction models that are fitted to properties of terrestrial nuclear observables largely fail to constrain the isovector part of the nuclear interaction. This, in turn, affects model predictions for properties of nuclear matter with large isospin asymmetry that are present in the core of neutron stars $[25,44,45]$. As a result, different neutron-star matter equation of state models emerge that give rise to very different neutron-star structure properties, such as masses and radii [23,26], moments of inertia [27,84], and tidal deformations [22,30]. Moreover, there is a possible degeneracy between the nuclear matter equation of state and models of gravity applied to describe the structure of neutron stars. While many studies have been devoted to break this degeneracy, it remains one of the outstanding problems to date $[5,13,20,38,49,55,69,79-$ $82,96,98]$.

In this contribution, we examine effects of the nuclear equation of state uncertainties simultaneously within the general theory of relativity (GR) and the scalar-tensor (ST) theory of gravity. For over a century, GR has been tested in many astrophysical scenarios and thus far its agreement with experiments and observations have been remarkable [82]. Nevertheless, most of the general relativistic tests have been performed in the weakfield regime [72]. Neutron stars exhibit a strong curvature, and it is not yet clear whether gravitational field of such compact objects is fully described by GR. Astrophysical observations may help pin down theories of

F. J. Fattoyev ( $ه)$

Department of Physics, Manhattan College, Riverdale, New York, NY 10471, USA

E-mail: ffattoyev01@manhattan.edu 
gravitation in the strong-field regime provided that the equation of state of dense nuclear matter is well known at all densities relevant to neutron stars. In this article, we show that future astrophysical and gravitational wave observations from low- and canonical-mass neutron star will constrain the equation of state of nuclear matter, whereas measurement of radii and tidal deformations of massive neutron stars will aid in putting further constraints on the ST theories of gravity.

In Sect. 2, we present the formalism of calculating the structure of neutron stars in the scalar-tensor theory of gravity. Next, in Sect. 3, we review the equation of state of neutron-star matter within the relativistic mean-field framework and discuss, in particular, the current uncertainties in the isovector sector of the nuclear interaction. In Sect. 4, we present our results for the neutron-star structure calculation in both GR and ST theory of gravity. We demonstrate that the equation of state of nuclear matter can be constrained using canonical- and low-mass neutron-star observations irrespective of the models of gravity used. We also show that once the equation of state is constrained, in conjunction with the massive neutron-star observations, one may then probe and limit the parameter space of the ST theory of gravity.

Throughout this paper, we adopt geometric units, $c=1=G$, where $c$ is the speed of light, and $G$ is the gravitational constant, respectively.

\section{Neutron-star structure in scalar-tensor theory of gravity}

The scalar-tensor theories of gravitation are one of the most natural generalizations of general relativity that date back to early 1950 s $[8,19,31,47,48]$. According to these theories, the gravitational force is also mediated by a scalar field, $\varphi$, in addition to the second-rank metric tensor, $g_{\mu \nu}$, present in general relativity. One can write the most general form of the action defining this theory as $[14,99]$

$$
S=\frac{1}{16 \pi} \int \mathrm{d}^{4} x \sqrt{-g_{*}}\left[R_{*}-2 g_{*}^{\mu \nu} \varphi_{, \mu} \varphi_{, \nu}-V(\varphi)\right]+S_{\text {matter }}\left[\psi_{\text {matter }} ; A^{2}(\varphi) g_{* \mu \nu}\right],
$$

where $R^{*} \equiv g_{*}^{\mu \nu} R_{\mu \nu}^{*}$ is the curvature scalar of the so-called "Einstein metric", $g_{* \mu \nu}$, describing the pure spin-2 excitations, whereas $\varphi$ is a long-range scalar field describing spin- 0 excitations. Here $V(\varphi)$ is the scalar field potential, $\psi_{\text {matter }}$ is a collective representation of all matter fields, and $S_{\text {matter }}$ is the corresponding action of the matter represented by $\psi_{\text {matter }}$, which in turn is coupled to the "Jordan-Fierz metric" $g_{\mu \nu}$ that is related to the "Einstein metric" through the conformal transformation:

$$
g_{\mu \nu} \equiv A^{2}(\varphi) g_{* \mu \nu}
$$

The field equations are easily formulated in the "Einstein metric"; however, all non-gravitational physical experiments measure quantities in the "Jordan-Fierz metric" and thus they are referred to as the "physical metric". From here on, we refer to them as the Einstein frame and the Jordan frame, respectively.

Taking variation of the action $S$ with respect to the metric $g_{* \mu \nu}$ and the scalar field $\varphi$, we find the set of field equations in the Einstein frame

$$
\begin{aligned}
& G_{* \mu \nu}=8 \pi T_{* \mu \nu}+2 \varphi_{, \mu} \varphi_{, \nu}-g_{* \mu \nu} g_{*}^{\alpha \beta} \varphi_{, \alpha} \varphi_{, \beta}-\frac{1}{2} V(\varphi) g_{* \mu \nu}, \\
& \square_{*} \varphi-\frac{1}{4} \frac{\mathrm{d} V(\varphi)}{\mathrm{d} \varphi}=-4 \pi \alpha(\varphi) T_{*} .
\end{aligned}
$$

Here again $T_{* \mu \nu}$ is the energy-momentum tensor in the Einstein frame, which is related to the physical energy-momentum tensor in Jordan frame $T_{\mu \nu}$ via

$$
T_{* \mu \nu}=A^{2}(\varphi) T_{\mu \nu}
$$

and $T_{*}$ and $\alpha(\varphi)$ are defined as

$$
\begin{aligned}
& T_{*} \equiv T_{* \mu}^{\mu}, \\
& \alpha(\varphi) \equiv \frac{\mathrm{d} \ln A(\varphi)}{\mathrm{d} \varphi} .
\end{aligned}
$$


The energy-momentum conservation can either be expressed as $\nabla_{\nu} T_{\mu}^{v}=0$ in the Jordan frame, or

$$
\nabla_{* \nu} T_{* \mu}^{\nu}=\alpha(\varphi) T_{*} \nabla_{* \mu} \varphi
$$

in the Einstein frame.

We consider the neutron star to be made of a perfect fluid. In this case, the energy density $\mathcal{E}$, the pressure $P$ and the 4-velocity $u_{\mu}$ in the two frames are related via the following relations:

$$
\begin{aligned}
& \mathcal{E}_{*}=A^{4}(\varphi) \mathcal{E}, \\
& P_{*}=A^{4}(\varphi) P, \\
& u_{* \mu}=A^{-1}(\varphi) u_{\mu},
\end{aligned}
$$

where the subscript asterisks denote quantities in the Einstein frame, as usual. The space-time metric describing an unperturbed, non-rotating, spherically symmetric neutron star can be written as

$$
\mathrm{d} s_{*}^{2}=-e^{2 v(r)} \mathrm{d} t^{2}+e^{2 \lambda(r)} r^{2}+r^{2}\left(\mathrm{~d} \theta^{2}+\sin ^{2} \mathrm{~d} \phi^{2}\right),
$$

where

$$
e^{-2 \lambda(r)}=1-\frac{2 M(r)}{r} \equiv N^{2}(r)
$$

and the function $v(r)$ will be calculated later. Here we also defined $N(r) \equiv(1-2 M(r) / r)^{1 / 2}$, for convenience. Using the space-time metric above, one can arrive at the dimensionally reduced field equations [99], which in turn can be cast into equations for hydrostatic structure:

$$
\begin{aligned}
\frac{\mathrm{d} M(r)}{\mathrm{d} r} & =4 \pi r^{2} A^{4}(\varphi) \mathcal{E}(r)+\frac{r^{2}}{2} N^{2}(r) \chi^{2}(r)+\frac{r^{2}}{4} V(\varphi), \\
\frac{\mathrm{d} P(r)}{\mathrm{d} r} & =-(\mathcal{E}(r)+P(r))\left(\frac{\mathrm{d} \nu(r)}{\mathrm{d} r}+\alpha(\varphi) \chi(r)\right), \\
\frac{\mathrm{d} \nu(r)}{\mathrm{d} r} & =\frac{M(r)}{r^{2} N^{2}(r)}+\frac{4 \pi r}{N^{2}(r)} A^{4}(\varphi) P(r)+\frac{1}{2} r \chi^{2}(r)-\frac{r}{4 N^{2}(r)} V(\varphi), \\
\frac{\mathrm{d} \chi(r)}{\mathrm{d} r} & =\left\{4 \pi A^{4}(\varphi)[\alpha(\varphi)(\mathcal{E}(r)-3 P(r))+r \chi(r)(\mathcal{E}(r)-P(r))]\right. \\
& \left.-\frac{2 \chi(r)}{r}\left(1-\frac{M(r)}{r}\right)+\frac{1}{2} r \chi(r) V(\varphi)+\frac{1}{4} \frac{\mathrm{d} V(\varphi)}{\mathrm{d} \varphi}\right\} N^{-2}(r),
\end{aligned}
$$

where we introduced

$$
\chi(r) \equiv \frac{\mathrm{d} \varphi}{\mathrm{d} r} .
$$

It is well known that predictions of scalar-tensor theories are physically equivalent to nonlinear modified gravity theories [58]. In particular, for the $R^{2}$-gravity, where the Ricci scalar is replaced with $f(R)=R+a R^{2}$ in the Einstein-Hilbert action, the explicit form of the potential $V(\varphi)$ can be written as

$$
V(\varphi)=\frac{1}{4 a}\left(1-e^{-2 \varphi} \sqrt{3}\right)^{2}, \quad \alpha(\varphi)=-\frac{1}{\sqrt{3}} .
$$

Next, following Ref. [14], we set $V(\varphi)=0$, and consider a coupling function of the form

$$
A(\varphi)=\exp \left(\alpha_{0} \varphi+\frac{1}{2} \beta_{0} \varphi^{2}\right)
$$

The coupling constants $\alpha_{0}$ and $\beta_{0}$ are real numbers. It was shown that measurement of the surface atomic line redshifts from neutron stars could be used as a direct test of strong-field gravity theories [17]. In particular, coupling constants with $\alpha_{0}=0$ and $\beta_{0}=-8$ were used. Over the past decade, significant improvements have been made in constraining these coupling constants. For example, solar-system experiments, binary-pulsar 
and pulsar-white dwarf timing observations put some of the most stringent constraints on these constants that conservatively can be written as $[14,33]$

$$
\begin{aligned}
& \alpha_{0}^{2}=\partial \ln A\left(\varphi_{0}\right) / \partial \varphi_{0} \lesssim 2 \times 10^{-5}, \\
& \beta_{0}=\partial^{2} \ln A\left(\varphi_{0}\right) / \partial \varphi_{0}^{2} \gtrsim-5 .
\end{aligned}
$$

On the other hand, it was first shown in Ref. [14] that predictions by models with $\beta_{0}>-4.35$ cannot in general be distinguished from the general relativistic results due to the so-called "spontaneous scalarization" effect. Notice that GR is automatically recovered in the limits of $\alpha_{0}=0$ and $\beta_{0}=0$, where the Eqs. (14-17) are reduced to the famous Tolman-Oppenheimer-Volkoff equations [65,92].

We solve the interior and the exterior problem simultaneously using the following natural boundary conditions in the center of the star:

$$
P(0)=P_{\mathrm{c}}, \quad \mathcal{E}(0)=\mathcal{E}(\mathrm{c}), \quad \chi(0)=0 .
$$

We also demand cosmologically flat solution at infinity to agree with the observation:

$$
\lim _{r \rightarrow \infty} v(r)=0, \quad \lim _{r \rightarrow \infty} \varphi(r)=0 .
$$

The only input required to integrate Eqs. (14-17) is the equation of state of neutron-star matter, $\mathcal{E}=\mathcal{E}(P)$, that is trivial in the case of exterior solution. Once an EOS is supplemented, for a given central pressure $P(0)=P_{\mathrm{c}}$, one can integrate them from the center of the star $r=0$, all the way up to $r \rightarrow \infty$.

The stellar coordinate radius is then determined by the condition in which the pressure vanishes, i.e. $P\left(r_{\mathrm{s}}\right)=0$, where $r_{\mathrm{s}}$ is the surface radius in the Einstein frame. The physical radius of a neutron star $R$ is then found in the Jordan frame through

$$
R=A\left(\varphi\left(r_{\mathrm{s}}\right)\right) r_{\mathrm{s}}
$$

The physical stellar mass $M$ as measured by an observer at infinity-also known as the Arnowitt-Deser-Misner (ADM) mass - matches with the coordinate mass since at infinity the coupling function approaches unity.

\section{Neutron-star matter equation of state}

The structure of neutron stars is sensitive to the equation of state of cold, fully catalyzed, and neutron-rich matter. The matter inside neutron stars span many orders of magnitude in density leading to rich and exotic phases in their interiors. In the outer crust of neutron stars, the matter is organized into a Coulomb lattice of neutron-rich nuclei embedded in a degenerate electron gas [6,75]. The nuclear composition in this region is solely determined by the masses of neutron-rich nuclei in the region of $26<Z \lesssim 40$ and the pressure support is primarily provided by the degenerate electrons. The equation of state for this region is, therefore, relatively well known [6,36]. In this work, we adopt the outer crust equation of state by Haensel, Zdunik and Dobaczewski [36]. As the density increases, the nuclei in the outer crust become more and more neutron rich. At a density of about $\rho \approx 4 \times 10^{11} \mathrm{~g} / \mathrm{cm}^{-3}$, the nuclei in the outer crust become so neutron rich that they can no longer hold additional neutrons, and neutrons start dripping out. This region defines the boundary between the outer and the inner crust.

The inner crust extends from the neutron-drip density up to about $\rho \approx 2 / 3 \rho_{0}$, where the uniformity in the system is restored. On the top layers of the inner crust, nucleons continue to cluster into a Coulomb crystal of neutron-rich nuclei embedded in a uniform electron gas, where, however, now the system is also in a chemical equilibrium with a superfluid neutron gas [70]. As the density continues to increase, the spherical nuclei start to deform in an effort to reduce the Coulomb repulsion. As a result, the inner crust exhibits complex and exotic structures that are collectively known as "nuclear pasta" [37,57,73], which emerge from a dynamical competition between the short-range nuclear attraction and the long-range Coulomb repulsion. Although significant progress has been made in simulating this exotic region [24,41-43], the equation of state for this region remains highly uncertain and must be inferred from theoretical calculations. While a detailed knowledge of the equation of state for this region is important for the interpretations of cooling observations [21], its impact on the bulk properties of neutron stars is minimal [71]. For this region, therefore, we resort to the equation of state provided by Negele and Vautherin [63].

The structure of neutron stars are mostly sensitive to the equation of the state of the core, and the crustcore transition properties. In particular, most of the mass of neutron stars are contained in the liquid core that comprises a region of the star with densities as low as one-third to as high as ten times nuclear matter 
saturation density $\rho_{0}$. For this region, we employ the equation of state generated from various refinements of relativistic mean-field model by Serot and Walecka $[76,77,95]$. For consistency, the transition density from the liquid core to the solid crust is computed using the same relativistic mean-field models, where it is done by searching for the critical density at which the uniform system becomes unstable to small amplitude density oscillations [11]. We would like to emphasize that the crust-core transition density (hence transition pressure) plays an important role in neutron star bulk properties with various models leading to crust thicknesses that differ by over $1 \mathrm{~km}$ and predicting significantly different crustal components of the moment of inertia that are important in interpreting observations of pulsar glitches [71].

The equation of state for the uniform liquid core is based on an interaction Lagrangian that has been accurately calibrated to a variety of ground-state properties of both finite nuclei and infinite nuclear matter. This model includes a nucleon field $(\psi)$, a scalar-isoscalar meson field $(\phi)$, a vector-isoscalar meson field $\left(V^{\mu}\right)$, and an isovector meson field $\left(b^{\mu}\right)$ [76,77]. The free Lagrangian density for this model is given by [23,27]

$$
\begin{aligned}
\mathcal{L}_{0}= & \bar{\psi}\left(i \gamma^{\mu} \partial_{\mu}-m_{b}\right) \psi+\frac{1}{2} \partial_{\mu} \phi \partial^{\mu} \phi-\frac{1}{2} m_{s}^{2} \phi^{2} \\
& -\frac{1}{4} F^{\mu \nu} F_{\mu \nu}+\frac{1}{2} m_{v}^{2} V^{\mu} V_{\mu}-\frac{1}{4} \mathbf{b}^{\mu \nu} \mathbf{b}_{\mu \nu}+\frac{1}{2} m_{\rho}^{2} \mathbf{b}^{\mu} \mathbf{b}_{\mu},
\end{aligned}
$$

where $F_{\mu \nu}$ and $\mathbf{b}_{\mu \nu}$ are the nuclear isoscalar and isovector field tensors, respectively,

$$
\begin{aligned}
& F_{\mu \nu}=\partial_{\mu} V_{\nu}-\partial_{\nu} V_{\mu}, \\
& \mathbf{b}_{\mu \nu}=\partial_{\mu} \mathbf{b}_{\nu}-\partial_{\nu} \mathbf{b}_{\mu},
\end{aligned}
$$

where the parameters $m_{b}, m_{s}, m_{v}$, and $m_{\rho}$ represent the nucleon and meson masses and may be treated as empirical constants. The interacting component of Lagrangian density can be written by the following expression $[62,76,77]$ :

$$
\mathcal{L}_{\text {int }}=\bar{\psi}\left[g_{\mathrm{s}} \phi-\left(g_{\mathrm{v}} V_{\mu}+\frac{g_{\rho}}{2} \boldsymbol{\tau} \cdot \mathbf{b}_{\mu}\right) \gamma^{\mu}\right] \psi-U\left(\phi, V^{\mu}, \mathbf{b}^{\mu}\right)
$$

that includes Yukawa couplings-with coupling parameters, $g_{\mathrm{s}}, g_{\mathrm{v}}$, and $g_{\rho}$ - between the nucleon and meson fields. The Lagrangian density is also supplemented by nonlinear meson interactions, $U\left(\phi, V^{\mu}, \mathbf{b}^{\mu}\right)$ that improve the phenomenological standing of the model:

$$
U\left(\phi, V^{\mu}, \mathbf{b}^{\mu}\right)=\frac{\kappa}{3 !}\left(g_{\mathrm{s}} \phi\right)^{3}+\frac{\lambda}{4 !}\left(g_{\mathrm{s}} \phi\right)^{4}-\frac{\zeta}{4 !}\left(g_{\mathrm{v}}^{2} V_{\mu} V^{\mu}\right)^{2}-\Lambda_{\mathrm{v}}\left(g_{\rho}^{2} \mathbf{b}_{\mu} \cdot \mathbf{b}^{\mu}\right)\left(g_{\mathrm{v}}^{2} V_{v} V^{\nu}\right)
$$

The details on the calibration procedure can be found in Refs. $[12,45,76,77,91]$ and references therein.

While the full complexity of the quantum system cannot be tackled exactly, the ground-state properties of the system may be computed in a mean-field approximation. In this approximation, all the meson fields are replaced by their classical expectation values and their solution can be readily obtained by solving the classical Euler-Lagrange equations of motion. The only remnant of quantum behavior is in the treatment of the nucleon field which emerges from a solution to the Dirac equation in the presence of appropriate scalar and vector potentials $[76,77]$. Following standard mean-field practices, the energy density of the system is given by the following expression:

$$
\begin{aligned}
\mathcal{E}(\rho, \alpha)= & \frac{1}{\pi^{2}} \int_{0}^{k_{\mathrm{F}}^{p}} k^{2} E_{k}^{*} \mathrm{~d} k+\frac{1}{\pi^{2}} \int_{0}^{k_{\mathrm{F}}^{n}} k^{2} E_{k}^{*} \mathrm{~d} k+\frac{1}{2} m_{s}^{2} \phi_{0}^{2}+\frac{\kappa}{3 !}\left(g_{\mathrm{s}} \phi_{0}\right)^{3}+\frac{\lambda}{4 !}\left(g_{\mathrm{s}} \phi_{0}\right)^{4} \\
& +\frac{1}{2} m_{v}^{2} V_{0}^{2}+\frac{\zeta}{8}\left(g_{\mathrm{v}} V_{0}\right)^{4}+\frac{1}{2} m_{\rho}^{2} b_{0}^{2}+3 \Lambda_{\mathrm{v}}\left(g_{\mathrm{v}} V_{0}\right)^{2}\left(g_{\rho} b_{0}\right)^{2}
\end{aligned}
$$

where $\rho$ is the baryon density of the system, $\alpha=\left(\rho_{\mathrm{n}}-\rho_{\mathrm{p}}\right) / \rho$ is the neutron-proton asymmetry, $E_{k}^{*}=$ $\sqrt{k^{2}+m_{\mathrm{b}}^{* 2}}, m_{\mathrm{b}}^{*}=m_{\mathrm{b}}-g_{\mathrm{s}} \phi_{0}$ is the effective nucleon mass, $k_{\mathrm{F}}^{p}\left(k_{\mathrm{F}}^{n}\right)$ is the proton (neutron) Fermi momentum. Since the mean-field approximation is thermodynamically consistent, the pressure of the system at zero temperature may be obtained either directly from the energy-momentum tensor or from the energy density and its first derivative [76,77], that is,

$$
P(\rho, \alpha)=\rho \frac{\partial \mathcal{E}(\rho, \alpha)}{\partial \rho}-\mathcal{E}(\rho, \alpha) .
$$


It is often useful to expand the energy per nucleon of the system in even powers of $\alpha$ :

$$
E / A(\rho, \alpha)-m_{b}=E_{\mathrm{SNM}}(\rho)+\alpha^{2} S(\rho)+\mathcal{O}\left(\alpha^{4}\right),
$$

where $E_{\mathrm{SNM}}(\rho)$ is the energy per nucleon of symmetric nuclear matter, whereas $S(\rho)$ is referred to as the nuclear symmetry energy, a quantity that represents the increase in the energy of the system as it departs from the symmetric limit of equal number of neutrons and protons [40,93].

Further, it is customary to characterize the behavior of both symmetric nuclear matter and the symmetry energy in terms of a few bulk parameters near nuclear saturation density $\rho_{0}$ :

$$
\begin{aligned}
& E_{\mathrm{SNM}}(\rho)=\varepsilon_{0}+\frac{1}{2} K x^{2}+\cdots, \\
& S(\rho)=J+L x+\frac{1}{2} K_{\mathrm{sym}} x^{2}+\cdots,
\end{aligned}
$$

where $x \equiv\left(\rho-\rho_{0}\right) / 3 \rho_{0}$ is a dimensionless parameter, $\varepsilon_{0}$ and $K$ represent the energy per nucleon and the incompressibility coefficient of symmetric nuclear matter, respectively, whereas $J, L$, and $K_{\text {sym }}$ are the magnitude, slope and curvature of the symmetry energy at saturation. The bulk parameters of symmetric nuclear matter are relatively well constrained $[22,28,28]$. On the other hand, the density dependence of the nuclear symmetry energy remains unconstrained due to lack of sensitive isovector nuclear probes [29,54]. In this contribution, we will primarily concentrate on the impact of variations of density slope of the symmetry energy $L$ that is closely related to the pressure of pure neutron matter at saturation density.

We assume the neutron-star matter to consist of neutrons, protons, electrons, and muons in chemical equilibrium. We do not consider any "exotic" degrees of freedom, such as hyperons, meson condensates, or quarks. The electrons and muons are assumed to behave as relativistic free Fermi gases (with $m_{e} \equiv 0$ ). The muons appear in the system only after the electronic Fermi momentum becomes equal to the muon rest mass. The total energy density and pressure of the star are obtained by adding up the nucleonic and leptonic contributions.

\section{Results}

In this work, we use the FSUGold2 parametrization that was introduced by Ref. [12] to specifically apply for both finite nuclei and neutron stars. Of particular importance to this study is the role of omega-meson self-interactions, as described by the parameter $\zeta$ in the interaction Lagrangian density, that is used to tune the equation of state at high density to reproduce the maximum mass of a neutron star. It was first shown by Müller and Serot that using different values of $\zeta$ one can reproduce the same observed nuclear matter properties at nuclear saturation, yet produce maximum neutron-star masses - using general relativity only-that differ by almost one solar mass [62]. For example, models with $\zeta=0$ predict the maximum neutron-star masses of about $2.8 M_{\odot}$. Note that this tuning primarily affects the equation of state of symmetric nuclear matter at high density, which is relevant to the core of neutron stars. On the other hand, by including the nonlinear coupling constant $\Lambda_{\mathrm{v}}$, Horowitz and Piekarewicz showed that one can modify the density dependence of the symmetry energy [45]. It was shown that tuning $\Lambda_{\mathrm{v}}$ provides a simple and efficient method of controlling the density dependence of symmetry energy without compromising the success of the model in reproducing welldetermined ground-state observables. The original FSUGold 2 model has a relatively stiff symmetry energy with the density slope of $L=112.8 \mathrm{MeV}$. Following the same method as outlined in Ref. [27], we obtain a family of "FSUGold2" parametrizations with $L=47,60,80,100 \mathrm{MeV}$. We emphasize that in doing so, predictions for properties of finite nuclei, such as the binding energies and charge radii of closed shell nuclei, remain intact. The maximum mass of a neutron star predicted by the original FSUGold2 with $\zeta=0.0256$ and using the general relativistic TOV equations is $M_{\max }=2.07 M_{\odot}$, which is consistent with the observations of highly precise measurements of two massive neutron stars made at the Green Bank Telescope [4,18]. Indeed, the maximum possible mass of a neutron star may not be very far from this value as was recently shown by Refs. [60,61,74], $M_{\max } \approx 2.17 M_{\text {Sun. }}$. This in turn suggests that the $\zeta$ parameter that controls the stiffness of the equation of state of symmetric nuclear matter is already well constrained, and future observations of maximum mass may put even tighter constraints.

On the other hand, the original FSUGold2 model predicts the corresponding general relativistic radius of a canonical 1.4 solar mass neutron star to be $R=14.11 \mathrm{~km}$. Unfortunately, direct determination of the neutron-star radii at present is not quite satisfactory. Early attempts by Özel and collaborators to determine 
simultaneously the mass and radius of three X-ray bursters resulted in stellar radii to be between 8 and 10 $\mathrm{km}$ [66]. Later, Steiner et al. supplemented Özel's study with additional neutron stars and concluded that the most probable radius of a $1.4 M_{\odot}$ lies in the range of 10.4-12.9 $\mathrm{km}[86,87]$. Nevertheless, this more conservative estimate has been put into question by Suleimanov et al., who used a more complete model of the neutron-star atmosphere and obtained a radius greater than $14 \mathrm{~km}$ for a single source studied [89]. Recognizing this situation and the many challenges posed by the study of X-ray bursters, Guillot et al. concentrated on the determination of stellar radii by studying quiescent low-mass X-ray binaries (qLMXB) in globular clusters. By explicitly stating all their assumptions, in particular the common radius assumption, they were able to determine a rather small neutron-star radius of $9.4 \pm 1.2 \mathrm{~km}[34,35]$. And recently, an improved precision over previous measurements was obtained by incorporating distance uncertainties to the globular cluster M13 that suggests the neutron-star radius to be $12.3_{-1.7}^{+1.9} \mathrm{~km}$ and/or $15.3_{-2.2}^{+2.4} \mathrm{~km}$ depending on the composition of the atmosphere being as $\mathrm{H}$ or $\mathrm{He}$, respectively [78].

Since the density slope of the symmetry energy $L$ is related to the pressure of pure neutron matter at saturation density, and the pressure of the neutron-rich matter is strongly correlated with the neutron-star radius $[9,28]$, we build a family of the FSUGold2 parametrizations using different values of $L$. While these parametrizations provide an accurate description of ground-state properties of finite nuclei, they also predict stellar radii that differ by over $1 \mathrm{~km}$. The uncertainties in the density dependence of the symmetry energy exhibited by the RMF models here broadly bracket the uncertainties stemming from various nuclear many-body models. This includes from non-relativistic mean-field Skyrme interactions to microscopic calculations of the equation of state that are based on the nucleon-nucleon interactions and consider other degrees of freedom such as pions, $\Delta$-resonances, and hyperons $[10,25,56,83]$. Note, however, that none of our parametrizations can produce neutron-star radii that are smaller than $12 \mathrm{~km}$, which remains one of the biggest challenges today $[26,46]$.

Fortunately, the prospects for precision neutron-star mass-radius measurements have never been better, especially with the upcoming Neutron Star Interior Composition Explorer (NICER) X-ray timing mission that can put some of the tightest constraints in the near future. On the other hand, the recent gravitational wave observation from a binary neutron-star merger [1] has already put some indirect constraint on the neutron-star radii. In particular, using the upper limit on the tidal deformability measurement determined by LIGO and Virgo Collaboration, Refs. [3,30,90] have significantly constrained the allowed EoS models, which in turn predicted a radius of a canonical neutron star to be smaller than about $14 \mathrm{~km}$. The tidal deformability is an intrinsic neutron-star property that describes the tendency of a neutron star to develop a mass quadrupole as a response to the tidal field induced by its companion [16,32]. The dimensionless tidal polarizability $\Lambda$ is defined as follows:

$$
\Lambda=\frac{2 k_{2}}{3}\left(\frac{R}{M}\right)^{5},
$$

where $k_{2}$ is the second Love number [7,15]. The tidal deformability is highly sensitive to the stellar radius, $\Lambda \sim R^{5}$ and, therefore, its measurement can be used as a proxy to constrain the neutron-star radius that has been notoriously difficult to measure in the past $[34,35,39,53,64,66,67,85,86,89]$.

In Table 1, we provide with our calculations for neutron-star masses and radii predicted by both general relativity and the scalar-tensor theory of gravity. Notice that the variations of the density slope of the symmetry energy as depicted in various $L$-values predict neutron-star radii that are different by over $1 \mathrm{~km}$. Nevertheless, both general relativity and scalar-tensor theory predict very similar stellar radii for most

Table 1 Predictions for the masses and radii of neutron stars in both general relativity and scalar-tensor theory of gravity using a family of FSUGold2 interaction whose isovector coupling constants are tuned to give different values of the density slope of the symmetry energy $L$

\begin{tabular}{lllllllll}
\hline$L(\mathrm{MeV})$ & $R_{1.4}^{\mathrm{GR}}(\mathrm{km})$ & $R_{1.4}^{\mathrm{ST}}(\mathrm{km})$ & $R_{2.0}^{\mathrm{GR}}(\mathrm{km})$ & $R_{2.0}^{\mathrm{ST}}(\mathrm{km})$ & $M_{\max }^{\mathrm{GR}}\left(M_{\odot}\right)$ & $M_{\max }^{\mathrm{ST}}\left(M_{\odot}\right)$ & $R_{\max }^{\mathrm{GR}}(\mathrm{km})$ & $R_{\max }^{\mathrm{ST}}(\mathrm{km})$ \\
\hline 47.0 & 12.77 & 12.75 & 11.99 & 12.66 & 2.027 & 2.112 & 11.55 & 12.20 \\
60.0 & 13.08 & 13.06 & 12.09 & 12.81 & 2.024 & 2.109 & 11.64 & 12.33 \\
80.0 & 13.42 & 13.41 & 12.25 & 12.97 & 2.025 & 2.110 & 11.78 & 12.46 \\
100.0 & 13.81 & 13.80 & 12.59 & 13.21 & 2.041 & 2.128 & 11.97 & 12.65 \\
112.8 & 14.11 & 14.11 & 12.96 & 13.46 & 2.073 & 2.159 & 12.14 & 12.84 \\
\hline
\end{tabular}

Here superscripts "GR" and "ST" stand for general relativity and scalar-tensor theory of gravity, respectively, and subscripts

"1.4", "2.0", and "max" stand for 1.4-, 2.0- and maximum mass stellar configurations 
neutron stars, unless their mass is close to two solar mass. This suggests that measurements of canonical neutron-star radii, in particular, would be unable to constrain the parameters of the scalar-tensor theory but would be extremely useful in constraining the equation of state of nuclear matter. Moreover, given that the density slope of the symmetry energy is relatively insensitive to the maximum stellar mass which mostly constrains the equation of state of symmetric nuclear matter at high densities, one can use radii measurements to place significant constraints primarily on the density dependence of the nuclear symmetry energy. For massive neutron stars, the radii predictions in two models of gravity start to deviate. This phenomenon of the so-called "spontaneous scalarization" was first observed by Damour and EspositoFarèse [16].

For completeness, in Fig. 1, we also show the full mass-versus-radii relation predicted in both GR and ST. Since recent measurements of tidal deformability suggested that neutron stars with radii $R \gtrsim 14 \mathrm{~km}$ may have already been ruled out $[2,3,30,59,61,90,94]$, in this figure, we do not display predictions from the original FSUGold2 parametrization. It is safe to say that given that multi-messenger era of gravitational wave astronomy and X-ray observations of neutron stars is in its infancy, future observations of neutron stars in mass-range of $M \lesssim 1.4 M_{\odot}$ will undoubtedly put tighter constraints on the nuclear equation of state (below $\rho \approx 2.5 \rho_{0}$ corresponding to the central density of a neutron star) but not on the coupling constants of the scalar-tensor theory.

It is particularly interesting to compare the radii of two-solar mass neutron stars predicted by both theory of gravitation. As an example, we use the soft equation of state with $L=47 \mathrm{MeV}$ and find that stellar radii differ by $5.6 \%$ (see Table 1). Since massive neutron stars are rarely found in nature-lots of theoretical work and sufficient observational data may be required to distinguish general relativity from the scalar-tensor theory using radii observations from massive stars alone-we note that this may not be the case if one considers tidal deformability measurements which scales as $R^{5}$. In this contribution, we did not directly calculate tidal deformabilities in the scalar-tensor theory that in addition to radii also depends on the tidal Love number $k_{2}$ [68]. It has been shown, however, that within the general relativistic framework $k_{2}$ is less sensitive to the radius of a neutron star, and the $R^{5}$ scaling behavior in tidal deformability is quite robust [30]. It is worth to point out that the ratio $\left(R_{2.0}^{\mathrm{ST}} / R_{2.0}^{G R}\right)^{5}=1.312$ suggests that the corresponding tidal deformabilities in two models may differ by over $30 \%$. We will explore this in more details in future work. This is intriguing because while mass-radii measurements of low-mass neutron stars will constrain the equation of state, tidal deformability measurements of massive stars would enable to test models of gravity in strong regime.

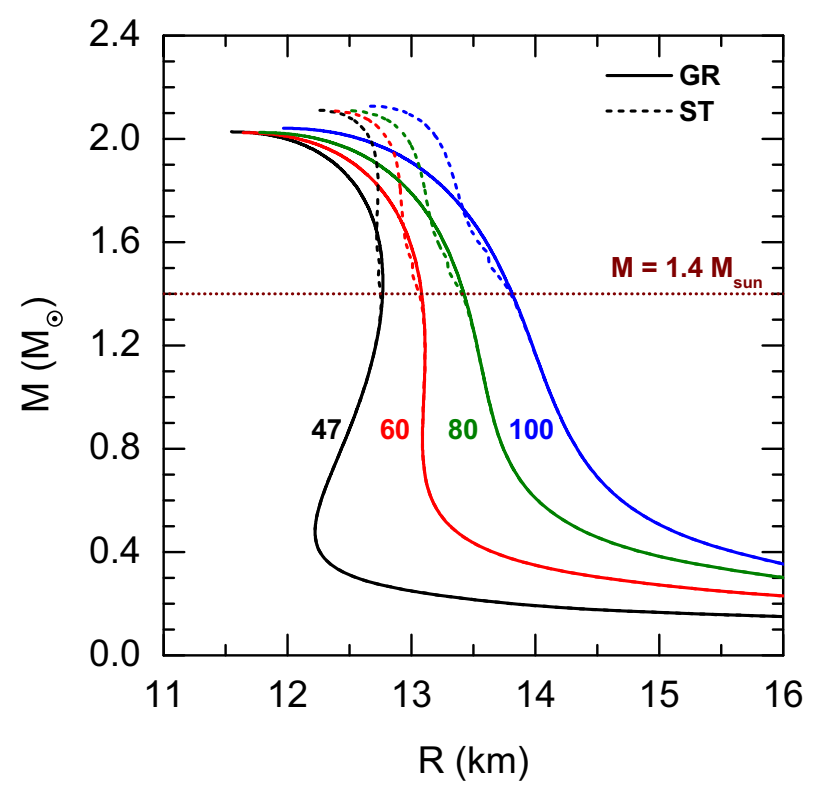

Fig. 1 The mass-versus-radius relation calculated using the family of FSUGold2 parametrizations considered in this work labeled with the corresponding $L$-values. Here $M$ stands for either general relativistic TOV-mass (solid lines) or the ADM-mass (dashed lines) predicted by the scalar-tensor theory. For the coupling constants of scalar-tensor theory an upper observational bounds of $\alpha_{0}=\sqrt{2.0} \times 10^{-5}$ and $\beta_{0}=-5.0$ are used 


\title{
5 Conclusions
}

We have examined bulk properties of neutron stars in general relativity and the scalar-tensor theory of gravity. In particular, we discussed the current uncertain role of the density dependence of the nuclear symmetry energy in the determination of the equation of state of neutron-star matter. Our analysis shows that future observations of neutron-star radii with masses $M \lesssim 1.4 M_{\odot}$ will enable to primarily constrain the equation of state of dense nuclear matter but not the coupling constants of the scalar-tensor theory of gravity. In particular, we have found that in conjunction with the maximum stellar mass measurements, this will lead to placing tight constraints on the density dependence of the nuclear symmetry energy.

Recently, it had been discussed that future observations of massive neutron stars may constrain the maximum sound velocity as well as the coupling parameter in the scalar-tensor theory of gravity [82]. While we confirm this result, we would also like to emphasize that once the equation of state is constrained using canonical- and low-mass neutron-star observations, and after a consensus has been reached on the upper limit of the neutron-star mass, only then one can place useful constraints on the coupling parameters of the scalar-tensor gravity. We would like to note that our work does not take into account the possibility that the core of neutron stars may have exotic degrees of freedom, such as hyperons, meson condensates, or quarks. In particular, the central density in neutron stars with $M \gtrsim 1.4 M_{\odot}$-for which spontaneous scalarization occurs-reaches nuclear densities of $\rho_{\mathrm{c}} \gtrsim 2.5 \rho_{0}$, where our knowledge of strong interaction is limited. Certainly there could still be a degeneracy between the scalar-tensor model of gravity and models of strong interaction at high densities. Much collaborative theoretical and observational efforts of both nuclear physics and gravitational physics community are, therefore, required on this front.

\begin{abstract}
Acknowledgements The author is grateful to Jorge Piekarewicz for providing the RPA code to calculate the crust-core transition properties, and to Xiao-Tao He, Charles J. Horowitz, Bao-An Li, and William G. Newton for many fruitful discussions. This work is supported in part by the U.S. Department of Energy Office of Science, Office of Nuclear Physics, under Awards DE-FG0287ER40365 and DE-SC0018083 (NUCLEI SciDAC-4 Collaboration) and by the Summer Grant from the Office of the Executive Vice President and Provost of Manhattan College.

Open Access This article is distributed under the terms of the Creative Commons Attribution 4.0 International License (http:// creativecommons.org/licenses/by/4.0/), which permits unrestricted use, distribution, and reproduction in any medium, provided you give appropriate credit to the original author(s) and the source, provide a link to the Creative Commons license, and indicate if changes were made.
\end{abstract}

\section{References}

1. Abbott, B.; et al.: (Virgo, LIGO Scientific), GW170817: observation of gravitational waves from a binary neutron star inspiral. Phys. Rev. Lett. 119, 161101 (2017). arXiv:1710.05832

2. Abbott, B.P.; et al.: (LIGO Scientific, Virgo), GW170817: measurements of neutron star radii and equation of state. Phys. Rev. Lett. 121, 161101 (2018). arXiv:1805.11581

3. Annala, E.; Gorda, T.; Kurkela, A.; Vuorinen, A.: Gravitational-wave constraints on the neutron-star-matter equation of state. Phys. Rev. Lett. 120, 172703 (2018). arXiv:1711.02644

4. Antoniadis, J.; Freire, P.C.; Wex, N.; Tauris, T.M.; Lynch, R.S.; et al.: A massive pulsar in a compact relativistic binary. Science 340, 6131 (2013). arXiv: 1304.6875

5. Arapoglu, A.S.; Deliduman, C.; Eksi, K.Y.: Constraints on perturbative f(R) gravity via neutron stars. JCAP 1107, 020 (2011). arXiv:1003.3179

6. Baym, G.; Pethick, C.; Sutherland, P.: The ground state of matter at high densities: equation of state and stellar models. Astrophys. J. 170, 299 (1971)

7. Binnington, T.; Poisson, E.: Relativistic theory of tidal Love numbers. Phys. Rev. D80, 084018 (2009). arXiv:0906.1366

8. Brans, C.; Dicke, R.H.: Mach's principle and a relativistic theory of gravitation. Phys. Rev. 124, 925 (1961) [142(1961)]

9. Brown, B.A.: Neutron radii in nuclei and the neutron equation of state. Phys. Rev. Lett. 85, 5296 (2000)

10. Cai, B.-J.; Fattoyev, F.J.; Li, B.-A.; Newton, W.G.: Critical density and impact of $\Delta(1232)$ resonance formation in neutron stars. Phys. Rev. C92, 015802 (2015). arXiv:1501.01680

11. Carriere, J.; Horowitz, C.J.; Piekarewicz, J.: Low mass neutron stars and the equation of state of dense matter. Astrophys. J. 593, 463 (2003). arXiv:nucl-th/0211015

12. Chen, W.-C.; Piekarewicz, J.: Building relativistic mean field models for finite nuclei and neutron stars. Phys. Rev. C90, 044305 (2014). arXiv: 1408.4159

13. Cooney, A.; DeDeo, S.; Psaltis, D.: Neutron stars in $\mathrm{f}(\mathrm{R})$ gravity with perturbative constraints. Phys. Rev. D82, 064033 (2010). arXiv:0910.5480

14. Damour, T.; Esposito-Farese, G.: Tensor-scalar gravity and binary pulsar experiments. Phys. Rev. D54, 1474 (1996). arXiv:gr-qc/9602056

15. Damour, T.; Nagar, A.; Villain, L.: Measurability of the tidal polarizability of neutron stars in late-inspiral gravitational-wave signals. Phys. Rev. D85, 123007 (2012). arXiv:1203.4352 
16. Damour, T.; Soffel, M.; Xu, C.-M.: General relativistic celestial mechanics. 2. Translational equations of motion. Phys. Rev. D45, 1017 (1992)

17. DeDeo, S.; Psaltis, D.: Towards new tests of strong-field gravity with measurements of surface atomic line redshifts from neutron stars. Phys. Rev. Lett. 90, 141101 (2003). arXiv:astro-ph/0302095

18. Demorest, P.B.; Pennucci, T.; Ransom, S.M.; Roberts, M.S.E.; Hessels, J.W.T.: Shapiro delay measurement of a two solar mass neutron star. Nature 467, 1081 (2010). arXiv: 1010.5788

19. Dicke, R.H.: Mach's principle and invariance under transformation of units. Phys. Rev. 125, 2163 (1962)

20. Eksi, K.Y.; Gungor, C.; Turkouglu, M.M.: What does a measurement of mass and/or radius of a neutron star constrain: equation of state or gravity? Phys. Rev. D89, 063003 (2014). arXiv:1402.0488

21. Fattoyev, F.J.; Brown, E.F.; Cumming, A.; Deibel, A.; Horowitz, C.J.; Li, B.-A.; Lin, Z.: Deep crustal heating by neutrinos from the surface of accreting neutron stars. Phys. Rev. C98, 025801 (2018). arXiv:1710.10367

22. Fattoyev, F.J.; Carvajal, J.; Newton, W.G.; Li, B.-A.: Constraining the high-density behavior of nuclear symmetry energy with the tidal polarizability of neutron stars. Phys. Rev. C87, 015806 (2013). arXiv:1210.3402

23. Fattoyev, F.J.; Horowitz, C.J.; Piekarewicz, J.; Shen, G.: Relativistic effective interaction for nuclei, giant resonances, and neutron stars. Phys. Rev. C82, 055803 (2010). arXiv:1008.3030

24. Fattoyev, F.J.; Horowitz, C.J.; Schuetrumpf, B.: Quantum nuclear pasta and nuclear symmetry energy. Phys. Rev. C95, 055804 (2017). arXiv:1703.01433

25. Fattoyev, F.J.; Newton, W.G.; Xu, J.; Li, B.-A.: Generic constraints on the relativistic mean-field and Skyrme-Hartree-Fock models from the pure neutron matter equation of state. Phys. Rev. C86, 025804 (2012). arXiv: 1205.0857

26. Fattoyev, F.J.; Piekarewicz, J.: Relativistic models of the neutron-star matter equation of state. Phys. Rev. C82, 025805 (2010). arXiv:1003.1298

27. Fattoyev, F.J.; Piekarewicz, J.: Sensitivity of the moment of inertia of neutron stars to the equation of state of neutron-rich matter. Phys. Rev. C82, 025810 (2010). arXiv:1006.3758

28. Fattoyev, F.J.; Piekarewicz, J.: Neutron skins and neutron stars. Phys. Rev. C86, 015802 (2012). arXiv:1203.4006

29. Fattoyev, F.J.; Piekarewicz, J.: Has a thick neutron skin in ${ }^{208} \mathrm{~Pb}$ been ruled out? Phys. Rev. Lett. 111, 162501 (2013). arXiv:1306.6034

30. Fattoyev, F.J.; Piekarewicz, J.; Horowitz, C.J.: Neutron skins and neutron stars in the multimessenger era. Phys. Rev. Lett. 120, 172702 (2018). arXiv:1711.06615.

31. Fierz, M.: On the physical interpretation of P. Jordan's extended theory of gravitation. Helv. Phys. Acta 29, 128 (1956)

32. Flanagan, E.E.; Hinderer, T.: Constraining neutron star tidal Love numbers with gravitational wave detectors. Phys. Rev. D77, 021502 (2008). arXiv:0709.1915

33. Freire, P.C.; Wex, N.; Esposito-Farese, G.; Verbiest, J.P.; Bailes, M. et al.: The relativistic pulsar-white dwarf binary PSR J1738+0333 II. The most stringent test of scalar-tensor gravity. Mon. Not. R. Astron. Soc. 423, 3328 (2012). arXiv: 1205.1450

34. Guillot, S.; Rutledge, R.E.: Rejecting proposed dense-matter equations of state with quiescent low-mass X-ray binaries. Astrophys. J. 796, L3 (2014). arXiv:1409.4306

35. Guillot, S.; Servillat, M.; Webb, N.A.; Rutledge, R.E.: Measurement of the radius of neutron stars with high S/N quiescent low-mass X-ray binaries in globular clusters. Astrophys. J. 772, 7 (2013). arXiv:1302.0023

36. Haensel, P.; Kutschera, M.; Proszynski, M.: Uncertainty in the saturation density of nuclear matter and neutron star models. Astron. Astrophys. 102, 299 (1981)

37. Hashimoto, M.; Seki, H.; Yamada, M.: Shape of nuclei in the crust of neutron star. Prog. Theor. Phys. 71, 320 (1984)

38. He, X.-T.; Fattoyev, F.J.; Li, B.-A.; Newton, W.G.: Impact of the equation-of-state-gravity degeneracy on constraining the nuclear symmetry energy from astrophysical observables. Phys. Rev. C91, 015810 (2015). arXiv:1408.0857

39. Heinke, C.O.; et al.: Improved mass and radius constraints for quiescent neutron stars in $\omega$ Cen and NGC 6397. Mon. Not. R. Astron. Soc. 444, 443 (2014). arXiv:1406.1497

40. Horowitz, C.; Brown, E.; Kim, Y.; Lynch, W.; Michaels, R.; et al.: A way forward in the study of the symmetry energy: experiment, theory, and observation. J. Phys. G41(2014), 093001 (2014). arXiv:1401.5839

41. Horowitz, C.J.; Perez-Garcia, M.A.; Berry, D.K.; Piekarewicz, J.: Dynamical response of the nuclear pasta in neutron star crusts. Phys. Rev. C72, 035801 (2005). arXiv:nucl-th/0508044

42. Horowitz, C.J.; Perez-Garcia, M.A.; Carriere, J.; Berry, D.K.; Piekarewicz, J.: Nonuniform neutron-rich matter and coherent neutrino scattering. Phys. Rev. C70, 065806 (2004). arXiv:astro-ph/0409296

43. Horowitz, C.J.; Perez-Garcia, M.A.; Piekarewicz, J.: Neutrino-pasta scattering: the opacity of nonuniform neutron-rich matter. Phys. Rev. C69, 045804 (2004). arXiv:astro-ph/0401079

44. Horowitz, C.J.; Piekarewicz, J.: The neutron radii of Lead and neutron stars. Phys. Rev. C64, 062802 (2001). arXiv:nucl-th/0108036.

45. Horowitz, C.J.; Piekarewicz, J.: Neutron star structure and the neutron radius of 208Pb. Phys. Rev. Lett. 86, 5647 (2001a). arXiv:astro-ph/0010227

46. Jiang, W.-Z.; Li, B.-A.; Fattoyev, F.J.: Small radii of neutron stars as an indication of novel in-medium effects. Eur. Phys. J. A51, 119 (2015). arXiv: 1509.02128

47. Jordan, P.: Formation of the stars and development of the universe. Nature 164, 637 (1949)

48. Jordan, P.: The present state of Dirac's cosmological hypothesis. Z. Phys. 157, 112 (1959)

49. Lasky, P.D.; Sotani, H.; Giannios, D.: Structure of neutron stars in tensor-vector-scalar theory. Phys. Rev. D78, 104019 (2008). arXiv:0811.2006

50. Lattimer, J.M.: The nuclear equation of state and neutron star masses. Ann. Rev. Nucl. Part. Sci. 62, 485 (2012). arXiv:1305.3510

51. Lattimer, J.M.; Prakash, M.: Neutron star structure and the equation of state. Astrophys. J. 550, 426 (2001). arXiv:astro-ph/0002232

52. Lattimer, J.M.; Prakash, M.: Neutron star observations: prognosis for equation of state constraints. Phys. Rept. 442, 109 (2007). arXiv:astro-ph/0612440 
53. Lattimer, J.M.; Steiner, A.W.: Neutron star masses and radii from quiescent low-mass X-ray binaries. Astrophys. J. 784, 123 (2014). arXiv:1305.3242

54. Li, B.-A.; Han, X.: Constraining the neutron-proton effective mass splitting using empirical constraints on the density dependence of nuclear symmetry energy around normal density. Phys. Lett. B727, 276 (2013). arXiv:1304.3368

55. Lin, W.; Li, B.-A.; Chen, L.-W.; Wen, D.-H.; Xu, J.: Breaking the EOS-gravity degeneracy with masses and pulsating frequencies of neutron stars. J. Phys. G41, 075203 (2014). arXiv:1311.3216

56. Lonardoni, D.; Lovato, A.; Gandolfi, S.; Pederiva, F.: Hyperon puzzle: hints from quantum Monte Carlo calculations. Phys. Rev. Lett. 114, 092301 (2015). arXiv: 1407.4448

57. Lorenz, C.P.; Ravenhall, D.G.; Pethick, C.J.: Neutron star crusts. Phys. Rev. Lett. 70, 379 (1993)

58. Magnano, G.; Sokolowski, L.M.: On physical equivalence between nonlinear gravity theories and a general relativistic selfgravitating scalar field. Phys. Rev. D50, 5039 (1994). arXiv:gr-qc/9312008

59. Malik, T.; Alam, N.; Fortin, M.; Providência, C.; Agrawal, B.K.; Jha, T.K.; Kumar, B.; Patra, S.K.: GW170817: constraining the nuclear matter equation of state from the neutron star tidal deformability. Phys. Rev. C98, 035804 (2018). arXiv: 1805.11963

60. Margalit, B.; Metzger, B.D.: Constraining the maximum mass of neutron stars from multi-messenger observations of GW170817. Astrophys. J. 850, L19 (2017). arXiv:1710.05938

61. Most, E.R.; Weih, L.R.; Rezzolla, L.; Schaffner-Bielich, J.: New constraints on radii and tidal deformabilities of neutron stars from GW170817. Phys. Rev. Lett. 120, 261103 (2018). arXiv:1803.00549

62. Mueller, H.; Serot, B.D.: Relativistic mean-field theory and the high-density nuclear equation of state. Nucl. Phys. A606, 508 (1996). arXiv:nucl-th/9603037

63. Negele, J.W.; Vautherin, D.: Neutron star matter at subnuclear densities. Nucl. Phys. A207, 298 (1973)

64. Nättilä, J.; Miller, M.C.; Steiner, A.W.; Kajava, J.J.E.; Suleimanov, V.F.; Poutanen, J.: Neutron star mass and radius measurements from atmospheric model fits to X-ray burst cooling tail spectra. Astron. Astrophys. 608, A31 (2017). arXiv: 1709.09120

65. Oppenheimer, J.R.; Volkoff, G.M.: On massive neutron cores. Phys. Rev. 55, 374 (1939)

66. Ozel, F.; Baym, G.; Guver, T.: Astrophysical measurement of the equation of state of neutron star matter. Phys. Rev. D82, 101301 (2010). arXiv: 1002.3153

67. Ozel, F.; Psaltis, D.; Guver, T.; Baym, G.; Heinke, C.; Guillot, S.: The dense matter equation of state from neutron star radius and mass measurements. Astrophys. J. 820, 28 (2016). arXiv:1505.05155

68. Pani, P.; Berti, E.: Slowly rotating neutron stars in scalar-tensor theories. Phys. Rev. D90, 024025 (2014). arXiv:1405.4547

69. Pani, P.; Berti, E.; Cardoso, V.; Read, J.: Compact stars in alternative theories of gravity. Einstein-Dilaton-Gauss-Bonnet gravity. Phys. Rev. D 84, 104035 (2011). arXiv:1109.0928

70. Piekarewicz, J.: The nuclear physics of neutron stars. AIP Conf. Proc. 1595, 76 (2014). arXiv:1311.7046

71. Piekarewicz, J.; Fattoyev, F.J.; Horowitz, C.J.: Pulsar glitches: the crust may be enough. Phys. Rev. C90, 015803 (2014). arXiv: 1404.2660

72. Psaltis, D.: Probes and tests of strong-field gravity with observations in the electromagnetic spectrum. Living Rev. Rel. 11, 9 (2008). arXiv:0806.1531

73. Ravenhall, D.G.; Pethick, C.J.; Wilson, J.R.: Structure of matter below nuclear saturation density. Phys. Rev. Lett. 50, 2066 (1983)

74. Rezzolla, L.; Most, E.R.; Weih, L.R.: Using gravitational-wave observations and quasi-universal relations to constrain the maximum mass of neutron stars. Astrophys. J. 852, L25 (2018). arXiv:1711.00314

75. Roca-Maza, X.; Piekarewicz, J.: Impact of the symmetry energy on the outer crust of non-accreting neutron stars. Phys. Rev. C78, 025807 (2008). arXiv:0805.2553

76. Serot, B.D.; Walecka, J.D.: The relativistic nuclear many body problem. Adv. Nucl. Phys. 16, 1 (1986)

77. Serot, B.D.; Walecka, J.D.: Recent progress in quantum hadrodynamics. Int. J. Mod. Phys. E6, 515 (1997). arXiv:nucl-th/9701058

78. Shaw, A.W.; Heinke, C.O.; Steiner, A.W.; Campana, S.; Cohn, H.N.; Ho, W.C.G.; Lugger, P.M.; Servillat, M.: The radius of the quiescent neutron star in the globular cluster M13. Mon. Not. R. Astron. Soc. 476, 4713 (2018). arXiv: 1803.00029

79. Sotani, H.: Slowly rotating relativistic stars in scalar-tensor gravity. Phys. Rev. D86, 124036 (2012). arXiv:1211.6986

80. Sotani, H.: Observational discrimination of Eddington-inspired Born-Infeld gravity from general relativity. Phys. Rev. D89, 104005 (2014). arXiv:1404.5369

81. Sotani, H.; Kokkotas, K.D.: Probing strong-field scalar-tensor gravity with gravitational wave asteroseismology. Phys. Rev. D70, 084026 (2004). arXiv:gr-qc/0409066

82. Sotani, H.; Kokkotas, K.D.: Maximum mass limit of neutron stars in scalar-tensor gravity. Phys. Rev. D95, 044032 (2017). arXiv:1702.00874.

83. Steiner, A.; Gandolfi, S.: Connecting neutron star observations to three-body forces in neutron matter and to the nuclear symmetry energy. Phys. Rev. Lett. 108, 081102 (2012). arXiv: 1110.4142

84. Steiner, A.W.; Gandolfi, S.; Fattoyev, F.J.; Newton, W.G.: Using neutron star observations to determine crust thicknesses, moments of inertia, and tidal deformabilities. Phys. Rev. C91, 015804 (2015). arXiv:1403.7546

85. Steiner, A.W.; Heinke, C.O.; Bogdanov, S.; Li, C.; Ho, W.C.G.; Bahramian, A.; Han, S.: Constraining the mass and radius of neutron stars in globular clusters. Mon. Not. R. Astron. Soc. 476, 421 (2018). arXiv: 1709.05013

86. Steiner, A.W.; Lattimer, J.M.; Brown, E.F.: The equation of state from observed masses and radii of neutron stars. Astrophys. J. 722, 33 (2010). arXiv:1005.0811

87. Steiner, A.W.; Lattimer, J.M.; Brown, E.F.: The neutron star mass-radius relation and the equation of state of dense matter. Astrophys. J. 765, L5 (2013). arXiv:1205.6871

88. Steiner, A.W.; Prakash, M.; Lattimer, J.M.; Ellis, P.J.: Isospin asymmetry in nuclei and neutron stars. Phys. Rept. 411, 325 (2005). arXiv:nucl-th/0410066

89. Suleimanov, V.; Poutanen, J.; Revnivtsev, M.; Werner, K.: Neutron star stiff equation of state derived from cooling phases of the X-ray burster 4U 1724-307. Astrophys. J. 742, 122 (2011). arXiv:1004.4871 
90. Tews, I.; Margueron, J.; Reddy, S.: Critical examination of constraints on the equation of state of dense matter obtained from GW170817. Phys. Rev. C98, 045804 (2018). arXiv:1804.02783

91. Todd, B.G.; Piekarewicz, J.: Relativistic mean-field study of neutron-rich nuclei. Phys. Rev. C67, 044317 (2003). arXiv:nucl-th/0301092

92. Tolman, R.C.: Static solutions of Einstein's field equations for spheres of fluid. Phys. Rev. 55, 364 (1939)

93. Tsang, M.; et al.: Constraints on the symmetry energy and neutron skins from experiments and theory. Phys. Rev. C86, 015803 (2012). arXiv:1204.0466

94. Tsang, C.Y.; Tsang, M.B.; Danielewicz, P.; Lynch, W.G., Fattoyev, F.J.: Constraining neutron-star equation of state using heavy-ion collisions (2018). arXiv:1807.06571

95. Walecka, J.D.: A theory of highly condensed matter. Ann. Phys. 83, 491 (1974)

96. Wen, D.-H.; Li, B.-A.; Chen, L.-W.: Super-soft symmetry energy encountering non-Newtonian gravity in neutron stars. Phys. Rev. Lett. 103, 211102 (2009). arXiv:0908.1922

97. Will, C.M.: The confrontation between general relativity and experiment. Living Rev. Rel. 17, 4 (2014). arXiv:1403.7377

98. Xu, J.; Li, B.-A.; Chen, L.-W.; Zheng, H.: Nuclear constraints on non-Newtonian gravity at femtometer scale. J. Phys. G40, 035107 (2013). arXiv:1203.2678

99. Yazadjiev, S.S.; Doneva, D.D.; Kokkotas, K.D.; Staykov, K.V.: Non-perturbative and self-consistent models of neutron stars in R-squared gravity. JCAP 1406, 003 (2014). arXiv:1402.4469

100. Yunes, N.; Siemens, X.: Gravitational-wave tests of general relativity with ground-based detectors and pulsar timing-arrays. Living Rev. Rel. 16, 9 (2013). arXiv:1304.3473

Publisher's Note Springer Nature remains neutral with regard to jurisdictional claims in published maps and institutional affiliations. 